\title{
SET STAR-MENGER AND SET STRONGLY STAR-MENGER SPACES
}

\author{
Luubiša D. R. KoČInaC*,c — Şukran KoncA** — Sumit SingH*** \\ (Communicated by David Buhagiar)
}

\begin{abstract}
Motivated by the Arhangel'skii "s-Lindelöf cardinal function" definition, Kočinac and Konca defined and studied set covering properties and set star covering properties. In this paper, we present results on the star covering properties called set star-Menger and set strongly star-Menger. We investigate the relationship among set star-Menger, set strongly star-Menger and other related properties and study the topological properties of set star-Menger and set strongly star-Menger properties.
\end{abstract} (c) 2022

Mathematical Institute Slovak Academy of Sciences

\section{Introduction and preliminaries}

In [1, Arhangel'skii defined a cardinal function $s L$, and spaces $X$ such that $s L(X)=\omega$ we call sLindelöf: a space $X$ is s-Lindelöf if for each subset $A$ of $X$ and each open cover $\mathcal{U}$ of $\bar{A}$ by open sets in $X$ there is a countable set $\mathcal{V} \subset \mathcal{U}$ such that $A \subset \overline{U \mathcal{V}}$. Motivated by this definition, Kočinac and Konca 14,15] (see also [6] for a similar study) considered new types of selective covering properties called set covering properties. In [14, the authors investigated the set-Menger, set-Hurewicz, setRothberger and other related (weaker) properties. Later on, Singh [19] and Kočinac [13] noticed that the properties set-Menger, set-Hurewicz and set-Rothberger are actually equvalent to Menger, Hurewicz and Rothberger properties, respectively.

In this paper, by using the star operator, we investigate the star versions of the set-Menger property called set star-Menger and set strongly star-Menger properties. Let us mention that set star-Menger spaces have been defined and considered the first time in 15. We discuss the relationships among the set star-Menger, set strongly star-Menger and other related properties. Further, we study topological properties of the set star-Menger and set strongly star-Menger properties.

If $A$ is a subset of a set $X$ and $\mathcal{F}$ is a collection of subsets of $X$, then the star of $A$ with respect to $\mathcal{F}$, denoted by $\operatorname{St}(A, \mathcal{F})$, is the set $\operatorname{St}(A, \mathcal{F})=\bigcup\{F \in \mathcal{F}: F \cap A \neq \emptyset\}$. We write $\operatorname{St}(x, \mathcal{F})$ instead of $\operatorname{St}(\{x\}, \mathcal{F})$.

Throughout the paper the following assumptions are considered:

(1) By "a space" we mean "a topological space".

(2) $\mathbb{N}$ denotes the set of natural numbers.

(3) An open cover $\mathcal{U}$ of a subset $A \subset X$ means elements of $\mathcal{U}$ are open in $X$ and $A \subset \cup \mathcal{U}=\cup\{U: U \in \mathcal{U}\}$.

2020 Mathematics Subject Classification: Primary 54D20; Secondary 54E35.

Keywords: Menger, star-Menger, strongly star-Menger, set star-Menger, set strongly star-Menger.

c Corresponding author. 
We first recall the classical notions of spaces which are used in this paper. The Menger covering property is a well-known covering property in topological spaces.

A space $X$ is said to have the Menger property [16] if for each sequence $\left(\mathcal{U}_{n}: n \in \mathbb{N}\right)$ of open covers of $X$, there is a sequence $\left(\mathcal{V}_{n}: n \in \mathbb{N}\right)$ such that for each $n \in \mathbb{N}, \mathcal{V}_{n}$ is a finite subset of $\mathcal{U}_{n}$ and $X=\bigcup_{n \in \mathbb{N}} \cup \mathcal{V}_{n}$.

Kočinac 10 11, introduced the star versions of the Menger covering property using star operator in the following way (for some articles related to the star versions of the classical selective covering properties see, for instance, $4,5,20,21$ ).

A space $X$ is said to have the star-Menger property (SM) [10 11] if for each sequence $\left(\mathcal{U}_{n}: n \in \mathbb{N}\right)$ of open covers of $X$, there is a sequence $\left(\mathcal{V}_{n}: n \in \mathbb{N}\right)$ such that for each $n \in \mathbb{N}, \mathcal{V}_{n}$ is a finite subset of $\mathcal{U}_{n}$ and $X=\bigcup_{n \in \mathbb{N}} \operatorname{St}\left(\cup \mathcal{V}_{n}, \mathcal{U}_{n}\right)$.

A space $X$ is said to be strongly star-Menger (SSM) [10,11] if for each sequence $\left(\mathcal{U}_{n}: n \in \mathbb{N}\right)$ of open covers of $X$, there is a sequence $\left(F_{n}: n \in \mathbb{N}\right)$ of finite subsets of $X$ such that $X=$ $\bigcup_{n \in \mathbb{N}} \operatorname{St}\left(F_{n}, \mathcal{U}_{n}\right)$.

Recently, Kočinac and Konca 14] defined set-Menger spaces and their weaker versions.

Definition $1([14])$. A space $X$ is said to have the set-Menger property (set-M) if for each nonempty set $A \subset X$ and each sequence $\left(\mathcal{U}_{n}: n \in \mathbb{N}\right)$ of collections of open sets in $X$ such that $\bar{A} \subset \cup \mathcal{U}_{n}$, there is a sequence $\left(\mathcal{V}_{n}: n \in \mathbb{N}\right)$ such that for each $n \in \mathbb{N}$, $\mathcal{V}_{n}$ is a finite subset of $\mathcal{U}_{n}$ and $A \subset \bigcup_{n \in \mathbb{N}} \cup \mathcal{V}_{n}$.

Remark 1. In [19 and 13], the authors noticed that set-Menger spaces are nothing but another way to define Menger spaces.

Definition 2 ( $2,7,17)$ ). A space $X$ is said to be:

(1) starcompact (SC) if for each open cover $\mathcal{U}$ of $X$ there is a finite subset $\mathcal{V}$ of $\mathcal{U}$ such that $X=\operatorname{St}(\cup \mathcal{V}, \mathcal{U})$

(2) strongly starcompact (SSC) if for each open cover $\mathcal{U}$ of $X$, there is a finite subset $F$ of $X$ such that $X=\operatorname{St}(F, \mathcal{U})$.

In a similar way, we define the following.

Definition 3. A space $X$ is said to be:

(1) set-starcompact (set-SC) if for each nonempty subset $A$ of $X$ and each open cover $\mathcal{U}$ of $\bar{A}$, there is a finite subset $\mathcal{V}$ of $\mathcal{U}$ such that $A=\operatorname{St}(\cup \mathcal{V}, \mathcal{U}) \cap A$;

(2) set strongly starcompact (set-SSC) if for each nonempty subset $A$ of $X$ and each open cover $\mathcal{U}$ of $\bar{A}$, there is a finite subset $F$ of $\bar{A}$ such that $A=\operatorname{St}(F, \mathcal{U}) \cap A$.

It is clear by the definition that every set strongly starcompact space is set-starcompact.

THEOREM 1.1. Every countably compact space is set strongly starcompact.

P r o of. Let $X$ be a countably compact space, $A$ a nonempty subset of $X$ and $\mathcal{U}$ a cover of $\bar{A}$ by open sets in $X$. As closed subsets of a countably compact space are also countably compact, $\bar{A}$ is countably compact. But any countably compact space is strongly starcompact 7 . Therefore, there is a finite set $F \subset \bar{A}$ such that $A \subset \bar{A} \subset \operatorname{St}(F, \mathcal{U})$, i.e. $X$ is set strongly starcompact.

Corollary 1.1.1. Every countably compact space is set-starcompact. 
In 7: Theorem 2.1.5] (see also [9]), it is proved that a strongly starcompact Hausorff space is countably compact. Since set strong starcompactness implies strong starcompactness, we have the following theorem.

THEOREM 1.2. If $X$ is a set strongly starcompact Hausdorff space, then $X$ is countably compact.

Recall that a collection $\mathcal{A} \subset P(\omega)$ is said to be almost disjoint if each set $A \in \mathcal{A}$ is infinite and the sets $A \cap B$ are finite for all distinct elements $A, B \in \mathcal{A}$. For an almost disjoint family $\mathcal{A}$, put $\psi(\mathcal{A})=\mathcal{A} \cup \omega$ and topologize $\psi(\mathcal{A})$ as follows: for each element $A \in \mathcal{A}$ and each finite set $F \subset \omega$, $\{A\} \cup(A \backslash F)$ is a basic open neighborhood of $A$ and the natural numbers are isolated. The spaces of this type are called Isbell-Mrówka $\psi$-spaces $[3,18]$ or $\psi(\mathcal{A})$ space.

Throughout the paper, notation and terminology mainly follow 8 . The cardinality of a set is denoted by $|A|$. Let $\omega$ denote the first infinite cardinal, $\omega_{1}$ the first uncountable cardinal, $\mathfrak{c}$ the cardinality of the set of real numbers. For a cardinal $\kappa, \kappa^{+}$denotes the smallest cardinal greater than $\kappa$. For each pair of ordinals $\alpha, \beta$ with $\alpha<\beta$, we write

$$
\begin{array}{ll}
{[\alpha, \beta)=\{\gamma: \alpha \leq \gamma<\beta\},} & (\alpha, \beta]=\{\gamma: \alpha<\gamma \leq \beta\}, \\
(\alpha, \beta)=\{\gamma: \alpha<\gamma<\beta\}, & {[\alpha, \beta]=\{\gamma: \alpha \leq \gamma \leq \beta\} .}
\end{array}
$$

As usual, a cardinal is an initial ordinal and an ordinal is the set of smaller ordinals. A cardinal is often viewed as a space with the usual order topology.

A subset $A$ of $X$ is said to be regular open if $A=\operatorname{Int}(\bar{A})$. A subset $B$ of $X$ is said to be regular closed if its complement is regular open or, equivalently, if $B=\overline{\operatorname{Int}(B)}$.

\section{Set star-Menger and set strongly star-Menger spaces}

In this section, we introduce set star-Menger and set strongly star-Menger spaces and give some examples showing the relationships between set star-Menger spaces and other related spaces. Some of these examples are known in the star selection principles theory and we discover some their additional properties.

Definition 4. A space $X$ is said to have:

(1) the set star-Menger property (shortly, set-SM property) if for each nonempty subset $A$ of $X$ and each sequence $\left(\mathcal{U}_{n}: n \in \mathbb{N}\right)$ of collections of open sets in $X$ such that $\bar{A} \subset \cup \mathcal{U}_{n}, n \in \mathbb{N}$, there is a sequence $\left(\mathcal{V}_{n}: n \in \mathbb{N}\right)$ such that for each $n \in \mathbb{N}, \mathcal{V}_{n}$ is a finite subset of $\mathcal{U}_{n}$ and $A \subset \bigcup_{n \in \mathbb{N}} \operatorname{St}\left(\bigcup \mathcal{V}_{n}, \mathcal{U}_{n}\right)$

(2) the set strongly star-Menger property (shortly, set-SSM property) if for each nonempty set $A \subset X$ and each sequence $\left(\mathcal{U}_{n}: n \in \mathbb{N}\right)$ of collections of open sets in $X$ such that $\bar{A} \subset \cup \mathcal{U}_{n}$, there is a sequence $\left(F_{n}: n \in \mathbb{N}\right)$ of finite subsets of $\bar{A}$ such that $A \subset \bigcup_{n \in \mathbb{N}} \operatorname{St}\left(F_{n}, \mathcal{U}_{n}\right)$.

Theorem 1.1 and the following lemma shows that the class of set strongly star-Menger spaces is big enough.

Lемма 2.1. Every Menger space is set strongly star-Menger.

Proof. Let $X$ be a Menger space. Let $A$ be any nonempty subset of $X$ and $\left(\mathcal{U}_{n}: n \in \mathbb{N}\right)$ be a sequence of collections of open sets in $X$ such that $\bar{A} \subset \cup \mathcal{U}_{n}$. Since closed subsets of Menger spaces are Menger, then $\bar{A}$ is Menger. Therefore, there exists a sequence $\left(\mathcal{V}_{n}: n \in \mathbb{N}\right)$ such that 
for each $n \in \mathbb{N}, \mathcal{V}_{n}$ is a finite subset of $\mathcal{U}_{n}$ and $\bar{A} \subset \bigcup_{n \in \mathbb{N}}\left(\cup \mathcal{V}_{n}\right)$. Choose $x_{V} \in \bar{A} \cap V$ for each $V \in \mathcal{V}_{n}$. For each $n \in \mathbb{N}$, let $F_{n}=\left\{x_{V}: V \in \mathcal{V}_{n}\right\}$. Then each $F_{n}$ is a finite subset of $\bar{A}$ and $A \subset \bar{A} \subset \bigcup_{n \in \mathbb{N}} \operatorname{St}\left(F_{n}, \mathcal{U}_{n}\right)$.

The following example shows that converse of Lemma 2.1 is not true.

Example 1. There exists a Tychonoff set strongly star-Menger space which is not Menger.

Pr o of. Let $X=\left[0, \omega_{1}\right)$. Then $X$ is a countably compact space, but not Lindelöf. Hence, $X$ is a set strongly star-Menger space which is not Menger.

We have the following diagram from the definitions and by Lemma 2.1. However, the converse of the implications may not be true as shown by examples below.

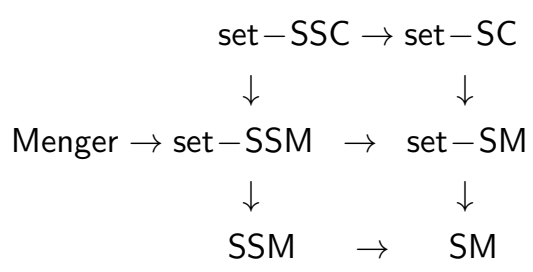

DIAGRAM 1.

We now show that vertical arrows between first and second lines of properties in Diagram 1 are not reversible.

Example 2. There exists a Tychonoff set star-Menger (resp., set strongly star-Menger) space which is not set-starcompact (resp., set strongly starcompact).

Proof. Let

$$
X=([0, \omega] \times[0, \omega]) \backslash\{\langle\omega, \omega\rangle\}
$$

be the subspace of the product space $[0, \omega] \times[0, \omega]$. Then $X$ is a Tychonoff space.

Since $X$ is countable, it is a Menger space. Since every Menger space is set strongly star-Menger and set star-Menger, we have that $X$ is set strongly star-Menger and set star-Menger.

Next, we show that $X$ is not set-starcompact. Let $A=\{\langle n, \omega\rangle: n \in \omega\}$ and for each $n \in \omega$, let

$$
U_{n}=\{n\} \times[n, \omega]
$$

Let us consider the open cover

$$
\mathcal{U}=\left\{U_{n}: n \in \omega\right\}
$$

of $A=\bar{A}$. To prove that $X$ is not set-starcompact, it is enough to show that there exists a point $a \in A$ such that $a \notin \operatorname{St}(\bigcup \mathcal{V}, \mathcal{U})$ for any finite subset $\mathcal{V}$ of $\mathcal{U}$. So, let $\mathcal{V}$ be a finite subset of $\mathcal{U}$. Then, there exists $n_{1} \in \omega$ such that $U_{n} \notin \mathcal{V}$ for each $n>n_{1}$. Let $a=\langle m, \omega\rangle$ with $m>n_{1}$. But $U_{m}$ is the only element of $\mathcal{U}$ containing $\langle m, \omega\rangle$. Thus $a=\langle m, \omega\rangle \notin \operatorname{St}(\bigcup \mathcal{V}, \mathcal{U})=\bigcup \mathcal{V}$, which shows that $X$ is not set-starcompact. It also follows that $X$ cannot be set strongly starcompact. This completes the proof.

We are going now to prove in the following two examples that vertical arrows between properties in the second and third lines of Diagram 1 are not reversible.

Example 3. There exists a Tychonoff star-Menger space which is not set star-Menger. 
P r o o f. Let $D(\mathfrak{c})=\left\{d_{\alpha}: \alpha<\mathfrak{c}\right\}$ be the discrete space of cardinality $\mathfrak{c}$, and let

$$
X=\left(\beta D(\mathfrak{c}) \times\left(\mathfrak{c}^{+}+1\right)\right) \backslash\left((\beta D(\mathfrak{c}) \backslash D(\mathfrak{c})) \times\left\{\mathfrak{c}^{+}\right\}\right)
$$

be the subspace of the product space $\beta D(\mathfrak{c}) \times\left(\mathfrak{c}^{+}+1\right)$, where $\beta D(\mathfrak{c})$ is the Stone-Čech compactification of $D(\mathfrak{c})$.

In 22. Example 2.2], Song shows that $X$ is a star-Menger space. Now we prove that $X$ is not set star-Menger.

Let $A=D(\mathfrak{c}) \times\left\{\mathfrak{c}^{+}\right\}$. Then $A$ is a closed subset of $X$. For each $\alpha<\mathfrak{c}$, let

$$
U_{\alpha}=\left\{d_{\alpha}\right\} \times\left(\mathfrak{c}^{+}+1\right) .
$$

Then $U_{\alpha}$ is open in $X$ by the construction of the topology of $\mathrm{X}$ and $U_{\alpha} \cap U_{\alpha^{\prime}}=\emptyset$ for $\alpha \neq \alpha^{\prime}$. For each $n \in \mathbb{N}$, let $\mathcal{U}_{n}=\left\{U_{\alpha}: \alpha<\mathfrak{c}\right\}$. Then $\left(\mathcal{U}_{n}: n \in \mathbb{N}\right)$ is a sequence of open covers of $\bar{A}$. It is enough to show that there exists a point $\left\langle d_{\beta}, \mathfrak{c}^{+}\right\rangle \in A$ such that

$$
\left\langle d_{\beta}, \mathfrak{c}^{+}\right\rangle \notin \operatorname{St}\left(\cup \mathcal{V}_{n}, \mathcal{U}_{n}\right) \text { for all } n \in \mathbb{N},
$$

for any sequence $\left(\mathcal{V}_{n}: n \in \mathbb{N}\right)$ such that $\mathcal{V}_{n}$ is a finite subset of $\mathcal{U}_{n}, n \in \mathbb{N}$. Let $\left(\mathcal{V}_{n}: n \in \mathbb{N}\right)$ be any sequence such that for each $n \in \mathbb{N}, \mathcal{V}_{n}$ is a finite subset of $\mathcal{U}_{n}$. For each $n \in \mathbb{N}, \mathcal{V}_{n}$ is finite, then there exists $\alpha_{n}<\mathfrak{c}$ such that $U_{\alpha} \notin \mathcal{V}_{n}$ for each $\alpha>\alpha_{n}$. Let

$$
\alpha^{\prime}=\sup \left\{\alpha_{n}: n \in \mathbb{N}\right\} \text {. }
$$

If we pick $\beta>\alpha^{\prime}$, then $U_{\beta} \notin \mathcal{V}_{n}$ for each $n \in \mathbb{N}$. Since $U_{\beta}$ is the only element of $\mathcal{U}_{n}$ containing the point $\left\langle d_{\beta}, \mathfrak{c}^{+}\right\rangle$for each $n \in \mathbb{N}$, then $\left\langle d_{\beta}, \mathfrak{c}^{+}\right\rangle \notin \operatorname{St}\left(\cup \mathcal{V}_{n}, \mathcal{U}_{n}\right)$ for all $n \in \mathbb{N}$, which shows that $X$ is not set star-Menger.

Example 4. Assuming $\omega_{1}<\mathfrak{d}=\mathfrak{c}$, there exists a Tychonoff pseudocompact strongly star-Menger space which is not set strongly star-Menger.

Proof. Let $X=\psi(\mathcal{A})$ be the Isbell-Mrówka space with $\mathcal{A}$ is maximal and $|\mathcal{A}|=\omega_{1}$. Then $X$ is strongly star-Menger Tychonoff pseudocompact space (see [3]). Now we prove that $X$ is not set strongly star-Menger. Let $A=\mathcal{A}=\left\{a_{\alpha}: \alpha<\omega_{1}\right\}$. Then $A$ is closed subset of $X$. For each $\alpha<\omega_{1}$, let $U_{\alpha}=\left\{a_{\alpha}\right\} \cup\left(a_{\alpha}\right)$. For each $n \in \mathbb{N}$, let $\mathcal{U}_{n}=\left\{U_{\alpha}: \alpha<\omega_{1}\right\}$. Then $\left(\mathcal{U}_{n}: n \in \mathbb{N}\right)$ is a sequence of open covers of $\bar{A}$. It is enough to show that there exists a point $a_{\beta} \in A$ such that

$$
a_{\beta} \notin \operatorname{St}\left(F_{n}, \mathcal{U}_{n}\right) \text { for all } n \in \mathbb{N},
$$

for any sequence $\left(F_{n}: n \in \mathbb{N}\right)$ of finite subsets of $\bar{A}$. Let $\left(F_{n}: n \in \mathbb{N}\right)$ be any sequence of finite subsets of $\bar{A}$. Then there exists $\alpha^{\prime}<\omega_{1}$ such that $U_{\alpha} \cap\left(\bigcup_{n \in \mathbb{N}} F_{n}\right)=\emptyset$, for each $\alpha>\alpha^{\prime}$. Pick $\beta>\alpha^{\prime}$, then $U_{\beta} \cap F_{n}=\emptyset$ for each $n \in \mathbb{N}$. Since $U_{\beta}$ is the only element of $\mathcal{U}_{n}$ containing the point $a_{\beta}$ for each $n \in \mathbb{N}$, then $a_{\beta} \notin \operatorname{St}\left(F_{n}, \mathcal{U}_{n}\right)$ for all $n \in \mathbb{N}$. It shows that $X$ is not set strongly star-Menger.

We now show that horizontal arrows between first and second lines of properties in Diagram 1 are not reversible.

Example 5. There exists a $T_{1}$ set star-Menger space which is not set strongly star-Menger.

Proof. Let $X=A \cup B$, where $A=\left\{a_{\alpha}: \alpha<\mathfrak{c}\right\}$ is any set with $|A|=\mathfrak{c}$ and $B$ is any countable set such that any element of $B$ is not in $A$. Topologize $X$ as follows: for each $a_{\alpha} \in A$ and each finite subset $F \subset B,\left\{a_{\alpha}\right\} \cup(B \backslash F)$ is a basic open neighborhood of $a_{\alpha}$, and each element of $B$ is isolated. Then $X$ is a $T_{1}$-space. 
First we show that $X$ is set star-Menger space. Let $C$ be any nonempty subset of $X$ and $\left(\mathcal{U}_{n}: n \in \mathbb{N}\right)$ be any sequence of open covers of $\bar{C}$. We have three possible cases.

Case (i): $C \subset A$.

Then for each $n \in \mathbb{N}$ and for each $a_{\alpha} \in C$, there exists $U_{\alpha, n} \in \mathcal{U}_{n}$ such that $a_{\alpha} \in U_{\alpha, n}$, i.e. we can find a finite set $F_{\alpha, n} \subset B$ such that $\left\{a_{\alpha}\right\} \cup\left(B \backslash F_{\alpha, n}\right) \subset U_{\alpha, n}$. Then, it is clear that for each $n \in \mathbb{N}$ and each $\beta<\mathfrak{c}$ with $\beta \neq \alpha, U_{\alpha, n} \cap U_{\beta, n} \neq \emptyset$. For each $n \in \mathbb{N}$, let $\mathcal{V}_{n}=\left\{U_{\alpha, n}\right\}$. Then each $\mathcal{V}_{n}$ is a finite subset of $\mathcal{U}_{n}$ and $C \subset \bigcup_{n \in \mathbb{N}} \operatorname{St}\left(\cup \mathcal{V}_{n}, \mathcal{U}_{n}\right)$.

Case (ii): $C \subset B$.

Since $B$ is countable, $B$ is set star Menger. Therefore, for each $n \in \mathbb{N}$ there is a finite subset $\mathcal{W}_{n}$ of $\mathcal{U}_{n}$ such that $C \subset \bigcup_{n \in \mathbb{N}} \operatorname{St}\left(\cup \mathcal{W}_{n}, \mathcal{U}_{n}\right)$.

Case (iii): $C \subset A \cup B$.

Let $C=C_{1} \cup C_{2}$, where $C_{1} \subset A$ and $C_{2} \subset B$. Choose $\mathcal{V}_{n} \subset \mathcal{U}_{n}$ and $\mathcal{W}_{n} \subset \mathcal{U}_{n}$ such that $C_{1} \subset \bigcup_{n \in \mathbb{N}} \operatorname{St}\left(\cup \mathcal{V}_{n}, \mathcal{U}_{n}\right)$ and $C_{2} \subset \bigcup_{n \in \mathbb{N}} \operatorname{St}\left(\cup \mathcal{W}_{n}, \mathcal{U}_{n}\right)$. Let $\mathcal{H}_{n}=\mathcal{V}_{n} \cup \mathcal{W}_{n}$. Then $\mathcal{H}_{n}$ is a finite subset of $\mathcal{U}_{n}$ and $C \subset \bigcup_{n \in \mathbb{N}} \operatorname{St}\left(\cup \mathcal{H}_{n}, \mathcal{U}_{n}\right)$.

So, we conclude that $X$ is a set star-Menger space.

Taking $A=\bar{A} \subset X$ and working quite similarly to the proof of Example 4 we easily prove that $X$ is not a set strongly star-Menger space.

It would be interesting to answer the following question.

Problem 1. Does there exist a Tychonoff set star-Menger space which is not set strongly starMenger?

Remark 2. (1) The space $X$ in Example 5 is a set-starcompact space but not set strongly starcompact. So, we have proved that the horizontal arrow in the first line of properties in Diagram 1 is not reversible.

(2) It is known that there are star-Menger spaces which are not strongly star-Menger (see, for example, [5, 12]) so that the horizontal arrow in the last line of Diagram 1 is not reversible.

In some classes of spaces certain properties from Diagram 1 coincide. Kočinac proved the following theorem in 10 .

TheOREM 2.1 ([10 Theorem 2.8]). In the class of paracompact Hausdorff spaces, Mengerness, star-Mengerness and strongly star-Mengerness coincide.

From Theorem 2.1 and Diagram 1 we get the following result.

THEOREM 2.2. If $X$ is a paracompact Hausdorff space, then the following statements are equivalent:

(1) $X$ is Menger;

(2) $X$ is set star-Menger;

(3) $X$ is set strongly star-Menger;

(4) $X$ is strongly star-Menger;

(5) $X$ is star-Menger. 
Remark 3. A space $X$ is said to be para-Lindelöf if every open cover $\mathcal{U}$ of $X$ has a locallycountable open refinement $\mathcal{V}$. In [5. Theorem 2.10] it was proved that in the class of regular para-Lindelöf spaces Mengerness, star-Mengerness and strongly star-Mengerness are equivalent. So, Theorem 2.2 can be extended to the class of regular para-Lindelöf spaces.

A space is said to be meta-Lindelöf if every open cover of it has a point-countable open refinement. Kočinac 10 obtained the following corollary.

Corollary 2.2.1 (10: Corollary 2.10]). A regular meta-Lindelöf strongly star-Menger space is Menger.

Corollary 2.2.1 and Diagram 1 give the following corollary.

COROLLARY 2.2.2. If $X$ is a regular meta-Lindelöf space, then the following statements are equivalent:

(1) $X$ is Menger;

(2) $X$ is set strongly star-Menger;

(3) $X$ is strongly star-Menger.

A space $X$ is metacompact if each open cover of $X$ has a point-finite open refinement. From [10. Theorem 2.4], we have the following corollary.

COROLLARY 2.2.3. Every set strongly star-Menger hereditarily metacompact space $X$ is a Menger space.

\section{Properties of set star-Menger and set strongly star-Menger spaces}

In this section, we study some topological properties of set star-Menger and set strongly starMenger spaces.

Observe that set star-Mengerness and set strong star-Mengerness are not hereditary properties. The ordinal space $\left[0, \omega_{1}\right)$ is a Hausdorff countably compact space, therefore it is set strongly starMenger. But its subspace $Y=\{\alpha+1: \alpha$ is a limit ordinal $\}$ is uncountable and discrete, so it cannot be set strongly star-Menger.

Example 5 shows that a closed subset of a set star-Menger space need not be set star-Menger. The space $X$ in that example is set star-Menger, but the set $A=\left\{a_{\alpha}: \alpha<\mathfrak{c}\right\} \subset X$ is not, since it is a discrete closed subset with cardinality $\mathfrak{c}$.

We have the following results about preservation of set star-Mengerness and set strong starMengerness by subspaces.

THeOREM 3.1. A clopen subspace of a set star-Menger space is also set star-Menger.

P r o of. Let $X$ be a set star-Menger space and $Y \subset X$ be a clopen subspace. Let $A$ be any subset of $Y$ and $\left(\mathcal{U}_{n}: n \in \mathbb{N}\right)$ be any sequence of collections of open sets in $\left(Y, \tau_{Y}\right)$ such that for each $n \in \mathbb{N}, \mathrm{Cl}_{Y}(A) \subset \cup \mathcal{U}_{n}$. Since $Y$ is open, then $\left(\mathcal{U}_{n}: n \in \mathbb{N}\right)$ is a sequence of collections of open sets in $X$, and since $Y$ is closed, $C l_{Y}(A)=C l_{X}(A)$. Applying the set star-Mengerness of $X$, there exists a sequence $\left(\mathcal{V}_{n}: n \in \mathbb{N}\right)$ such that for each $n \in \mathbb{N}, \mathcal{V}_{n}$ is a finite subset of $\mathcal{U}_{n}$ and $A \subset \bigcup_{n \in \mathbb{N}} \operatorname{St}\left(\cup \mathcal{V}_{n}, \mathcal{U}_{n}\right)$. Hence $Y$ is set star-Menger. 
LJUBIŠA D. R. KOČINAC — ȘUKRAN KONCA — SUMIT SINGH

On the lines of Theorem 3.1, we can similarly prove the following theorem.

THEOREM 3.2. A clopen subspace of a set strongly star-Menger space is also set strongly starMenger.

We now consider (non)preservation of set star-Mengerness and set strong star-Mengerness under some sorts of mappings.

TheOREM 3.3. A continuous image of a set star-Menger space is set star-Menger.

Proof. Let $X$ be a set star-Menger space and $f: X \rightarrow Y$ a continuous mapping from $X$ onto $Y$. Let $B$ be any subset of $Y$ and $\left(\mathcal{V}_{n}: n \in \mathbb{N}\right)$ be a sequence of open covers of $\bar{B}$. Set $A=f^{\leftarrow}(B)$. Since $f$ is continuous, for each $n \in \mathbb{N}, \mathcal{U}_{n}=\left\{f^{\leftarrow}(V): V \in \mathcal{V}_{n}\right\}$ is a collection of open sets in $X$ with

$$
\bar{A}=\overline{f^{\leftarrow}(B)} \subset f^{\leftarrow}(\bar{B}) \subset f^{\leftarrow}\left(\cup \mathcal{V}_{n}\right)=\cup \mathcal{U}_{n} .
$$

As $X$ is set star-Menger, there exists a sequence $\left(\mathcal{U}_{n}^{\prime}: n \in \mathbb{N}\right)$ such that for each $n \in \mathbb{N}, \mathcal{U}_{n}^{\prime}$ is a finite subset of $\mathcal{U}_{n}$ and,

$$
A \subset \bigcup_{n \in \mathbb{N}} \operatorname{St}\left(\cup \mathcal{U}_{n}^{\prime}, \mathcal{U}_{n}\right)
$$

Let $\mathcal{V}_{n}^{\prime}=\left\{V: f^{\leftarrow}(V) \in \mathcal{U}_{n}^{\prime}\right\}$. Then for each $n \in \mathbb{N}, \mathcal{V}_{n}^{\prime}$ is a finite subset of $\mathcal{V}_{n}$ and

$$
\begin{aligned}
B & =f(A) \subset f\left(\bigcup_{n \in \mathbb{N}} \operatorname{St}\left(\cup \mathcal{U}_{n}^{\prime}, \mathcal{U}_{n}\right)\right) \subset \bigcup_{n \in \mathbb{N}} \operatorname{St}\left(\cup f\left(\left\{f^{\leftarrow}(V): V \in \mathcal{V}_{n}^{\prime}\right\}\right), \mathcal{V}_{n}\right) \\
& =\bigcup_{n \in \mathbb{N}} \operatorname{St}\left(\cup \mathcal{V}_{n}^{\prime}, \mathcal{V}_{n}\right) .
\end{aligned}
$$

Thus $Y$ is a set star-Menger space.

We can prove the following theorem similarly to the proof of Theorem 3.3 .

TheOREM 3.4. A continuous image of a set strongly star-Menger space is set strongly star-Menger.

Now we give a positive result on preimages of set strongly star-Menger spaces. For this we need a new concept defined as follows. We call a space $X$ nearly set stongly star-Menger if for each $A \subset X$ and each sequence $\left(\mathcal{U}_{n}: n \in \mathbb{N}\right)$ of open covers of $X$ there is a sequence $\left(F_{n}: n \in \mathbb{N}\right)$ of finite subsets of $X$ such that $A \subset \bigcup_{n \in \mathbb{N}} \operatorname{St}\left(F_{n}, \mathcal{U}_{n}\right)$.

THEOREM 3.5. Let $f: X \rightarrow Y$ be an open and closed, finite-to-one continuous mapping from a space $X$ onto a set strongly star-Menger space $Y$. Then $X$ is nearly set strongly star-Menger.

Proof. Let $A \subset X$ be any nonempty set and $\left(\mathcal{U}_{n}: n \in \mathbb{N}\right)$ be a sequence of open covers of $X$. Then $B=f(A)$ is a subset of $Y$. Let $y \in \bar{B}$. Then $f^{\leftarrow}(y)$ is a finite subset of $X$, and thus for each $n \in \mathbb{N}$, there is a finite subset $\mathcal{U}_{n, y}$ of $\mathcal{U}_{n}$ such that $f^{\leftarrow}(y) \subset \cup \mathcal{U}_{n, y}$ and $U \cap f^{\leftarrow}(y) \neq \emptyset$ for each $U \in \mathcal{U}_{n, y}$. Since $f$ is closed, there exists an open neighborhood $V_{n, y}$ of $y$ in $Y$ such that $f^{\leftarrow}\left(V_{n, y}\right) \subset \cup\left\{U: U \in \mathcal{U}_{n, y}\right\}$. Since $f$ is open, we can assume that

$$
V_{n, y} \subset \cap\left\{f(U): U \in \mathcal{U}_{n, y}\right\} .
$$

Now, for each $n \in \mathbb{N}, \mathcal{V}_{n}=\left\{V_{n, y}: y \in \bar{B}\right\}$ is an open cover of $\bar{B}$. Since $Y$ is set strongly star-Menger, there exist a sequence $\left(F_{n}: n \in \mathbb{N}\right)$ of finite subsets of $\bar{B}$ such that

$$
B \subset \bigcup_{n \in \mathbb{N}} \operatorname{St}\left(F_{n}, \mathcal{V}_{n}\right)
$$


Since $f$ is finite-to-one, the sequence $\left(f^{\leftarrow}\left(F_{n}\right): n \in \mathbb{N}\right)$ is a sequence of finite subsets of $X$. We prove

$$
A \subset \bigcup_{n \in \mathbb{N}} \operatorname{St}\left(f^{\leftarrow}\left(F_{n}\right), \mathcal{U}_{n}\right),
$$

Let $x \in A$. Then, there exists $n \in \mathbb{N}$ and $y \in \bar{B}$ such that $f(x) \in V_{n, y}$ and $V_{n, y} \cap F_{n} \neq \emptyset$. Since

$$
x \in f^{\leftarrow}\left(V_{n, y}\right) \subset \bigcup\left\{U: U \in \mathcal{U}_{n, y}\right\}
$$

we can choose $U \in \mathcal{U}_{n, y}$ with $x \in U$. Then $V_{n, y} \subset f(U)$. Thus $U \cap f^{\leftarrow}\left(F_{n}\right) \neq \emptyset$. Hence $x \in \operatorname{St}\left(f^{\leftarrow}\left(F_{n}\right), \mathcal{U}_{n}\right)$. It follows that

$$
A \subset \bigcup_{n \in \mathbb{N}} \operatorname{St}\left(f^{\leftarrow}\left(F_{n}\right), \mathcal{U}_{n}\right) .
$$

Thus $X$ is nearly set strongly star-Menger.

Following the above definition of nearly set strongly star-Menger spaces, we will call a space $X$ nearly set star-Menger if for each $A \subset X$ and each sequence $\left(\mathcal{U}_{n}: n \in \mathbb{N}\right)$ of open covers of $X$ there is a sequence $\left(\mathcal{V}_{n}: n \in \mathbb{N}\right)$ such that for each $n, \mathcal{V}_{n}$ is a finite subset of $\mathcal{U}_{n}$ and $A \subset \bigcup_{n \in \mathbb{N}} \operatorname{St}\left(\cup \mathcal{V}_{n}, \mathcal{U}_{n}\right)$.

Similarly to the proof of Theorem 3.5, with necessary small modifications, we can prove the following.

THEOREM 3.6. If $f: X \rightarrow Y$ is an open perfect mapping and $Y$ is a set star-Menger space, then $X$ is nearly set star-Menger.

The product of two set star-Menger spaces need not be set star-Menger. In fact, the following well-known example (see 8, 24]) shows that the product of two countably compact (hence set strongly star-Menger) spaces need not be set star-Menger (hence not set strongly star-Menger). Song [23. Example 2.12] proved that the product $X \times Y$ is not star-Menger. Thus $X \times Y$ is not set star-Menger. Here we give a rough proof for the sake of completeness.

Example 6. There exist two countably compact spaces $X$ and $Y$ such that $X \times Y$ is not set star-Menger.

Proof. Let $D(\mathfrak{c})$ be the discrete space of cardinality $\mathfrak{c}$. We can define $X=\bigcup_{\alpha<\omega_{1}} E_{\alpha}, Y=\bigcup_{\alpha<\omega_{1}} F_{\alpha}$, where $E_{\alpha}$ and $F_{\alpha}$ are subsets of $\beta D(\mathfrak{c})$ defined inductively so that the following three conditions are satisfied:

(1) $E_{\alpha} \cap F_{\beta}=D(\mathfrak{c})$ if $\alpha \neq \beta$;

(2) $\left|E_{\alpha}\right| \leq \mathfrak{c}$ and $\left|F_{\alpha}\right| \leq \mathfrak{c}$;

(3) every infinite subset of $E_{\alpha}$ (resp., $F_{\alpha}$ ) has an accumulation point in $E_{\alpha+1}$ (resp., $F_{\alpha+1}$ ).

Those sets $E_{\alpha}$ and $F_{\alpha}$ are well-defined since every infinite closed set in $\beta D(\mathfrak{c})$ has cardinality $2^{\mathfrak{c}}$ (see [24]). The diagonal $\{\langle d, d\rangle: d \in D(\mathfrak{c})\}$ is a discrete open and closed subset of $X \times Y$ of cardinality $\mathfrak{c}$ so that it is not set star-Menger. Thus $X \times Y$ is not set star-Menger, since, by Theorem 3.1. open and closed subsets of set star-Menger spaces are set star-Menger.

In the following example, initially considered in [7 Example 3.3.3], we show that the product of a countably compact (hence, set star-Menger) space and a Lindelöf space need not be set starMenger.

Example 7. There exist a countably compact (hence, set star-Menger) space $X$ and a Lindelöf space $Y$ such that $X \times Y$ is not set star-Menger. 
P r o o f. Let $X=\left[0, \omega_{1}\right)$ be equipped with the order topology, and let $Y=\left[0, \omega_{1}\right]$ with the following topology. Each point $\alpha<\omega_{1}$ is isolated, and a set $U$ containing $\omega_{1}$ is open if and only if $Y \backslash U$ is countable. Then, $X$ is countably compact and $Y$ is Lindelöf. By [23 Example 2.13], $X \times Y$ is not star-Menger. Thus it cannot be set star-Menger.

Above results suggest the following problem.

Problem 2. Is the product of a set strongly star-Menger (set star-Menger) space with a compact space set strongly star-Menger (set star-Menger)?

\section{Concluding remarks}

This paper deals with two set star properties. Some further investigations on this kind of star covering properties (which we began to study) may include similar properties of Hurewicz-type and Rothberger-type star selection principles, as well as some weaker forms of set-SM, set-SSM spaces and their Hurewicz-type and Rothberger-type versions. We give here definitions of these properties.

Definition 5. A space $X$ is said to be:

(1) set star-Hurewicz (resp., set strongly star-Hurewicz) if for each $A \subset X$ and each sequence $\left(\mathcal{U}_{n}: n \in \mathbb{N}\right)$ of open covers of $\bar{A}$ there is a sequence $\left(\mathcal{V}_{n}: n \in \mathbb{N}\right.$ ) (resp., a sequence $\left(F_{n}: n \in \mathbb{N}\right)$ ) such that $\mathcal{V}_{n}$ is a finite subset of $\mathcal{U}_{n}$ (resp., $F_{n}$ is a finite subset of $\bar{A}$ ) for each $n \in \mathbb{N}$, and each $x \in A$ belongs to all but finitely many sets $\operatorname{St}\left(\cup \mathcal{V}_{n}, \mathcal{U}_{n}\right)$ (resp., to all but finitely many sets $\left.\operatorname{St}\left(F_{n}, \mathcal{U}_{n}\right)\right)$;

(2) set star-Rothberger (resp., set strongly star-Rothberger) if for each $A \subset X$ and each sequence $\left(\mathcal{U}_{n} ; n \in \mathbb{N}\right)$ of collections of open sets in $X$ such that $\bar{A} \subset \cup \mathcal{U}_{n}$, there is a sequence $\left(U_{n}\right.$ : $n \in \mathbb{N}$ ) (resp., a sequence $\left(x_{n}: n \in \mathbb{N}\right)$ ) such that $U_{n} \in \mathcal{U}_{n}$ (resp., $x_{n} \in \bar{A}$ ) for each $n \in \mathbb{N}$ and $A \subset \bigcup_{n \in \mathbb{N}} \operatorname{St}\left(U_{n}, \mathcal{U}_{n}\right)$ (resp., $\left.A \subset \bigcup_{n \in \mathbb{N}} \operatorname{St}\left(x_{n}, \mathcal{U}_{n}\right)\right)$.

Definition 6. A space $X$ is said to be:

(1) weakly set star-Menger (almost set star-Menger, faintly set star-Menger) if for each $A \subset X$ and each sequence $\left(\mathcal{U}_{n}: n \in \mathbb{N}\right)$ of open covers of $\bar{A}$ by open sets in $X$, there is a sequence $\left(\mathcal{V}_{n}: n \in \mathbb{N}\right)$ such that $\mathcal{V}_{n}$ is a finite subset of $\mathcal{U}_{n}$ for each $n \in \mathbb{N}$, and $A \subset \overline{\bigcup_{n \in \mathbb{N}} \operatorname{St}\left(\cup \mathcal{V}_{n}, \mathcal{U}_{n}\right)}$, $A \subset \bigcup_{n \in \mathbb{N}} \overline{\operatorname{St}\left(\cup \mathcal{V}_{n}, \mathcal{U}_{n}\right)}\left(A \subset \bigcup_{n \in \mathbb{N}} \operatorname{St}\left(\overline{\cup \mathcal{V}_{n}}, \mathcal{U}_{n}\right)\right) ;$

(2) weakly set strongly star-Menger (almost set strongly star-Menger) if for each $A \subset X$ and each sequence $\left(\mathcal{U}_{n}: n \in \mathbb{N}\right)$ of open covers of $\bar{A}$ there is a sequence $\left(F_{n}: n \in \mathbb{N}\right)$ of finite subsets of $\bar{A}$ such that $A \subset \overline{\bigcup_{n \in \mathbb{N}} \operatorname{St}\left(F_{n}, \mathcal{U}_{n}\right)}\left(A \subset \bigcup_{n \in \mathbb{N}} \overline{\operatorname{St}\left(F_{n}, \mathcal{U}_{n}\right)}\right)$.

In a similar way we define weaker forms of Hurewicz-type and Rothberger-type properties.

Acknowledgement. The authors are grateful to the referees for their careful reading of the paper and for a number of comments and remarks which improved the exposition of the paper. 


\section{SET STAR-MENGER AND SET STRONGLY STAR-MENGER SPACES}

\section{REFERENCES}

[1] ARHANGEL'SKII, A. V.: A generic theorem in the theory of cardinal invariants of topological spaces, Comment. Math. Univ. Carolin. 36 (1995), 303-325.

[2] Alas, O. T.-JUNQUEIRA, L. R.-WILSON, R. G.: Countability and star covering properties, Topology Appl. 158 (2011), 620-626.

[3] BonanZIngA, M.-MAtVeev, M. V.: Some covering properties for $\psi$-spaces, Mat. Vesnik 61 (2009), 3-11.

[4] BONAnZINGA, M.-CAMMAROTO, F.-KOČINAC, LJ. D. R.-MATVEEV, M. V.: On weaker forms of Menger, Rothberger and Hurewicz properties, Mat. Vesnik 61 (2009) 13-23.

[5] CASAS-DE LA ROSA, J.-GARCIA-BALAN, S. A.-SZEPTYCKI, P. J.: Some star and strongly star selection principles, Topology Appl. 258 (2019), 572-587.

[6] DI MAIO, G.-KOČINAC, LJ. D. R.: A note on quasi-Menger and similar spaces, Topology Appl. 179 (2015), $148-155$.

[7] VAN DOUWEn, E. K.-REED, G. K.-ROSCOE, A. W.-TREE, I. J.: Star covering properties, Topology Appl. 39 (1991), 71-103.

[8] ENGELKING, R.: General Topology, PWN, Warszawa, 1977.

[9] FLEISCHMAN, W. M.: A new extension of countable compactness, Fund. Math. 67 (1971), 1-7.

[10] KOČINAC, LJ. D. R.: Star-Menger and relalted spaces, Publ. Math. Debrecen 55 (1999), 421-431.

[11] KOČINAC, LJ. D. R.: Star-Menger and related spaces II, Filomat 13 (1999), 129-140.

[12] KOČINAC, LJ. D. R.: Variations of classical selection principles: an overview, Quaest. Math. 37 (2020), $1121-1153$.

[13] KOČINAC, LJ. D. R.: Addendum to: "Variations of classical selection principles: an overview", Quaest. Math. 44 (2022), 1281-1282.

[14] KOČINAC, LJ. D. R.-KONCA, Ş.: Set-Menger and related properties, Topology Appl. 275 (2020), Art. No. 106996.

[15] KONCA, Ş.-KOČINAC, LJ. D. R.: Set-star Menger and related spaces, Abstract Book VI ICRAPAM 2019 (Istanbul, Turkey, June 12-15, 2019), p. 49.

[16] MENGER, K.: Einige Überdeckungssätze der Punktmengenlehre, Sitzungsberischte Abt. 2a, Math., Astron., Phys., Meteor. Mech. (Wiener Akademie, Wien) 133 (1924), 421-444.

[17] MatVeEV, M. V.: A survey on star-covering properties, Topology Atlas, Preprint No. 330, 1998.

[18] MRÓWKA, S.: On completely regular spaces, Fund. Math. 41 (1954), 105-106.

[19] SINGH, S.: Remarks on set-Menger and related properties, Topology Appl. 280 (2020), Article No. 107278.

[20] SONG, Y. K.: Remarks on star-Hurewicz spaces, Bull. Polish Acad. Sci. Math. 61 (2013), 247-255.

[21] SONG, Y. K.: Remarks on strongly star-Hurewicz spaces, Filomat 27 (2013), 1127-1131.

[22] SONG, Y. K.: Remarks on star-Menger spaces, Houston J. Math. 40 (2014), 917-925.

[23] SONG, Y. K.: Remarks on star-Menger spaces II, Houston J. Math. 41 (2015), 357-366.

[24] WALKER, R. C.: The Stone-Čech Compactification, Berlin, 1974 
LJUBIŠA D. R. KOČINAC — ȘUKRAN KONCA - SUMIT SINGH

Received 28. 9. 2020

Accepted 31. 1. 2021
* Faculty of Sciences and Mathematics

University of Niš

Višegradska 33

$18000 \mathrm{Nis̆}$

SERBIA

E-mail: lkocinac@gmail.com

** Department of Fundamental Sciences/Mathematics Faculty of Engineering and Architecture

Izmir Bakircay University

35665 Izmir

TURKEY

E-mail: sukran.konca@bakircay.edu.tr

*** Department of Mathematics

University of Delhi

110007 New Delhi

INDIA

E-mail: sumitkumar405@gmail.com 